\title{
Genomics and biochemistry investigation on the metabolic pathway of milled wood and alkali lignin-derived aromatic metabolites of Comamonas serinivorans SP-35
}

Daochen Zhu ${ }^{1,2^{*}}$ (D) Haibing Si ${ }^{1}$, Peipei Zhang ${ }^{1}$, Alei Geng ${ }^{1}$, Weimin Zhang ${ }^{2}$, Bin Yang ${ }^{3}$, Wei-Jun Qian ${ }^{4}$, Murillo Gabriel ${ }^{1}$ and Jianzhong Sun ${ }^{1 *}$

\begin{abstract}
Background: The efficient depolymerization and utilization of lignin are one of the most important goals for the renewable use of lignocelluloses. The degradation and complete mineralization of lignin by bacteria represent a key step for carbon recycling in land ecosystems as well. However, many aspects of this process remain unclear, for example, the complex network of metabolic pathways involved in the degradation of lignin and the catabolic pathway of intermediate aromatic metabolites. To address these subjects, we characterized the deconstruction and mineralization of lignin with milled wood lignin (MWL, the most representative molecule of lignin in its native state) and alkali lignin (AL), and elucidated metabolic pathways of their intermediate metabolites by a bacterium named Comamonas serinivorans SP-35.

Results: The degradation rate of MWL reached $30.9 \%$, and its particle size range was decreased from 6 to $30 \mu \mathrm{m}$ to 2-4 $\mu \mathrm{m}$ - when cultured with $\mathrm{C}$. serinivorans SP35 over 7 days. FTIR analysis showed that the $\mathrm{C}-\mathrm{C}$ and $\mathrm{C}-\mathrm{O}-\mathrm{C}$ bonds between the phenyl propane structures of lignin were oxidized and cleaved and the side chain structure was modified. More than twenty intermediate aromatic metabolites were identified in the MWL and AL cultures based on GCMS analysis. Through genome sequencing and annotation, and from GC-MS analysis, 93 genes encoding 33 enzymes and 5 regulatory factors that may be involved in lignin degradation were identified and more than nine metabolic pathways of lignin and its intermediates were predicted. Of particular note is that the metabolic pathway to form the powerful antioxidant 3,4-dihydroxyphenylglycol is described for the first time in bacteria.

Conclusion: Elucidation of the $\beta$-aryl ether cleavage pathway in the strain SP-35 indicates that the $\beta$-aryl ether catabolic system is not only present in the family of Sphingomonadaceae, but also other species of bacteria kingdom. These newly elucidated catabolic pathways of lignin in strain SP-35 and the enzymes responsible for them provide exciting biotechnological opportunities for lignin valorization in future.
\end{abstract}

Keywords: Comamonas serinivorans SP-35, Metabolic pathway, Lignin, Whole genome sequencing, Aromatic metabolites

\footnotetext{
*Correspondence: dczhucn@hotmail.com; jzsun1002@hotmail.com

${ }^{1}$ Biofuels Institute, School of the Environment and Safety Engineering,

Jiangsu University, Zhenjiang, Jiangsu, China

Full list of author information is available at the end of the article
} 


\section{Background}

Lignin is the most abundant natural aromatic compound on the planet and is a promising source of renewable aromatic building blocks, such as sinapyl alcohol, coniferyl alcohol and p-coumaryl alcohol. Because lignin is a highly cross-linked polymer formed by the polymerization of 4-hydroxyphenylpropanoid monomer units such as $p$-hydroxyphenyl $(\mathrm{H})$, guaiacyl $(\mathrm{G})$ and syringyl $(\mathrm{S})$ units and linked by ether or carbon-carbon bonds, its complicated 3-dimensional polymer network structure has a highly recalcitrant nature. However, the biosphere has already developed catabolic pathways for lignin since its introduction into the global carbon cycle [1]. Among the lignin degraders, fungi have been already extensively studied in recent decades; the high oxidative capacity of some fungal enzymes is able to attack lignin and some lignin fragments. The enzymes involved in lignin degradation in fungi include lignin peroxidases (LiP), manganese peroxidases $(\mathrm{MnP})$, versatile peroxidases $(\mathrm{VP})$, dye decolorizing peroxidases (DyPs) and copper-containing oxidative laccases (Lacc). In comparison to fungi, lignin degradation by bacteria has been less studied. However, DyPs have been identified as an important class of oxidases involved in the bacterial degradation of lignin. It has been reported that Lacc and DyPs, were observed as oxidases in lignin degrading bacteria, but without LiP, $\mathrm{MnP}$ and VP [2, 3]. Bacteria do not possess the typical peroxidases that fungi employ for lignin degradation, which suggests that the ligninolytic enzymatic system of bacteria might be different from that of fungus.

Recently, an abundant number of species of lignin degrading bacteria have been identified and studied in detail because of their broad growth range and tolerance to extreme environments. For example, Bacillus ligniniphilus L1 is a halotolerant and alkaliphilic bacterium which is able to grow in a broad range of temperatures $\left(10-50{ }^{\circ} \mathrm{C}\right)$ and $\mathrm{pH}(7-11$, with the optimal $\mathrm{pH} 9$ for lignin degradation), has great potential for the treatment of high-salt and high-pH wastewater from papermaking, printing, and dyeing [4]. In addition, bacteria were not only able to depolymerize lignin but also played a major role in the mineralization of lignin-derived heterogeneous aromatic compounds [5]. There are multiple metabolic pathways of aromatic compounds that are involved in the mineralization of lignin after lignin depolymerization in bacteria, such as $\beta$-aryl ether, biphenyl, protocatechuate, catechol and gentisate pathways. [6, 7]. Dioxygenases play an important role in these pathways, which cleave carbon-carbon bonds of aromatic rings to produce ring-opened products that enter central carbon metabolism via $\beta$-ketoadipate pathway ( $\beta$-KAP) [8]. However, there are still many metabolic pathways that are unknown because hundreds of lignin derivatives have been identified from lignin [9]. Therefore, elucidating these metabolic pathways in bacteria is beneficial for producing high-value aromatic compounds from lignin through genetic manipulations such as knocking out the genes of key step enzymes. So far, most of the literature about the bacterial degradation of lignin used alkali lignin (AL) or kraft lignin as the substrate. However, during the production of $\mathrm{AL}$, sodium hydroxide was used, and the hydrolysis and condensation reactions destroyed the cross-linked structure of lignin, forming different molecule sizes. Compared to AL, milled wood lignin (MWL) is more suitable for the investigation of lignin degradation by bacteria, as it has been typically considered to be the most representative molecule of lignin in its native state $[10,11]$. Therefore, it is important to characterize the degradation of lignin by bacteria using MWL as the substrate, examine the intermediate metabolites, and clarify their metabolic pathways during the process of lignin depolymerization and mineralization. The objective of this study was to demonstrate the depolymerization of MWL and AL to produce low-molecularweight aromatic metabolic intermediates by the strain Comamonas serinivorans SP-35 DSM $26136^{\mathrm{T}}$, as well as the reconstruction of the catabolic pathways of those lignin-derived aromatic compounds by gas chromatography-mass spectrometry analysis and whole-genome sequencing and annotation.

\section{Methods}

\section{Strain and media}

Comamonas serinivorans SP-35 DSM $26136^{\mathrm{T}}$ was isolated from compost by our lab and identified as a new species of genus Comamonas [12]. It was routinely cultured in Luria broth (LB) medium containing $(\mathrm{g} / \mathrm{L}): 10.0$ Bactopeptone (Difco Laboratories, Detroit, MI, USA); 5.0 yeast extract (Difco Laboratories); $5.0 \mathrm{NaCl}$. The lignin incubation medium (LM) used in this study was as follows $(\mathrm{g} / \mathrm{L}): \mathrm{K}_{2} \mathrm{HPO}_{4}, 1.0 ; \mathrm{MgSO}_{4}, 0.2 ; \mathrm{CaCl}_{2}, 0.05$; $\mathrm{FeSO}_{4}, 0.05 ; \mathrm{MnSO}_{4}, 0.02 ; \mathrm{KH}_{2} \mathrm{PO}_{4}, 1.0 ;\left(\mathrm{NH}_{4}\right)_{2} \mathrm{SO}_{4}, 2.0$; and $0.5 \mathrm{~g}$ MWL or AL (CAS 8068-05-1, Catalog number 370959, Sigma-Aldrich, St. Louis. Mo) as carbon source.

\section{Milled wood lignin preparation}

The isolation and purification of MWL were performed as described before [13]. The corncobs (collected from a farm from Yuncheng County, China) were dried at $50{ }^{\circ} \mathrm{C}$ for $12 \mathrm{~h}$ and milled to a size under 40 meshes. Then, the samples were extracted using ethanol for $4 \mathrm{~h}$ and a benzene/ethanol $(2: 1, \mathrm{v} / \mathrm{v})$ solution for $8 \mathrm{~h}$ using a Soxhlet extractor and dried. Then, the samples were ground $(600 \mathrm{rpm})$ for $72 \mathrm{~h}$ in a vibratory ball mill with stainless steel balls and toluene. Afterwards, the ball-milled wood samples were extracted twice with dioxane/water 
$(2 \times 200 \mathrm{~mL} ; 96: 4, \mathrm{v} / \mathrm{v})$ for $24 \mathrm{~h}$ under a nitrogen atmosphere, and the supernatant was freeze-dried to obtain the crude MWL. The crude MWL was dissolved in acetic acid/water solution (9:1, v/v), dropped into deionized water overnight, and then centrifuged and freeze-dried. The crude lignin dissolved in dichloroethane/ethanol $(2: 1, \mathrm{v} / \mathrm{v})$ was added to diethyl ether dropwise, and the un-dissolved fraction was separated by centrifugation and freeze-dried to obtain the purified MWL.

\section{Growth of strain SP-35 and remove of chemical oxygen demand}

Cells of SP-35 were inoculated in LB medium at $30{ }^{\circ} \mathrm{C}$ for $18 \mathrm{~h}$, and then the pellets were collected by centrifugation and washed twice with a potassium phosphate buffer $(100 \mathrm{mM})$ to remove the residual medium and re-suspended in $0.9 \%(\mathrm{v} / \mathrm{v}) \mathrm{NaCl}$ solution. For growth culture, $1 \mathrm{~mL}$ of re-suspended cells were inoculated in $100 \mathrm{~mL}$ of LM medium with $0.05 \mathrm{~g}$ of MWL or glucose as the carbon source or without carbon source as control and incubated in the shaker at $30{ }^{\circ} \mathrm{C}, 220 \mathrm{rpm}$. For the measurement of chemical oxygen demand (COD), supernatant was collected by the centrifugation of culture at $10,000 \mathrm{rpm}$ for $5 \mathrm{~min}$. The dichromate method (standard methods, Section 5220D, Eaton et al. [14]) was used to determine COD [14]. All measurements were conducted in triplicate.

\section{Scanning electron microscopy analysis}

For scanning electron microscopy (SEM) analyses, the samples were centrifuged and the supernatants were freeze-dried. Afterwards, the samples were mounted on aluminum stubs, coated with a gold-palladium alloy, and examined with scanning electron microscopy (SEM, JSM-7001F, Japan).

\section{Fourier-transform infrared spectrometer spectra analysis}

Fourier-transform infrared spectrometer (FTIR) spectra analysis was performed with a Bruker Tensor 27 spectrometer (Bruker Optics, Bullerica, MA), 0.001 g ground sample was mixed with $0.1 \mathrm{~g} \mathrm{KBr}$ and analyzed in the range of $4000-400 \mathrm{~cm}^{-1}$. The absorbance between 250 and $400 \mathrm{~nm}$ was measured.

\section{Gas chromatography-mass spectrometry analysis}

For gas chromatography-mass spectrometry (GC-MS) analysis, samples were prepared as described previously [4]. For the silylation procedure, $100 \mu \mathrm{L}$ dioxane and 10 $\mu \mathrm{L}$ pyridine were added into samples and vortexed in glass tubes followed by silylation, which was performed with $50 \mu \mathrm{L}$ of trimethylsilyl [BSTFA (N, O-bis (trimethylsilyl) trifluoroacetamide) and TMCS (trimethylchlorosilane)], Sigma-Aldrich, (Sigma-Aldrich, St. Louis. MO).
The mixture was heated in a water bath at $80{ }^{\circ} \mathrm{C}$ for $45 \mathrm{~min}$ with periodic shaking to dissolve the residues. GC-MS (AntoSystem XL GC-TurboMass; Perkin-Elmer, Waltham, MA, USA) analysis was carried out with PE$5 \mathrm{MS}$ capillary column $(20 \mathrm{~m} \times 0.18 \mathrm{~mm}$ internal diameter, $0.18 \mathrm{~mm}$ film thickness). The injection volume was 1 $\mu \mathrm{L}$ at a carrier gas flow of $1 \mathrm{~mL} \mathrm{~min}{ }^{-1}$ of helium. The column temperature program was $50{ }^{\circ} \mathrm{C}(5 \mathrm{~min}) ; 50-300{ }^{\circ} \mathrm{C}$ (10 ${ }^{\circ} \mathrm{C} \mathrm{min}^{-1}$, holding time: $5 \mathrm{~min}$ ). The transfer line and the ion source temperatures were maintained at 200 and $250{ }^{\circ} \mathrm{C}$. A solvent delay of 3.0 min was selected. In the full-scan mode, electron ionization mass spectra in the range of $30-550(\mathrm{~m} / \mathrm{z})$ were recorded at an electron energy of $70 \mathrm{eV}$. All standard monomeric phenolic compounds $(1 \mathrm{mg})$ were derivatized and chromatograph analyzed as above. To identify the low molecular weight lignin-related compounds as trimethylsilyl (TMS) derivatives derived from bacterial treatment, their mass spectra were compared with that of the data of the GC-MS spectral library (Wiley, NIST) available in the instrument and by comparing the retention time with those of some authentic compounds available.

\section{Genome sequencing and analysis of Comamonas serinivorans SP-35}

The genomic DNA of strain SP-35 was extracted using a Wizard Genomic DNA Purification Kit (Promega, Madison, WI) and whole-genome sequencing was performed by the Majorbio Company (Shanghai) using the PacBio RS II platform and done using the single molecule real time (SMRT) sequencing. A SMRT bell sequencing library with a 7-8 kb insert size was prepared using the PacBio SMRTbell Template Prep Kit 1.0. Afterwards, the raw data was assembled with the assistance of a Celera assembler and polished by Quiver tool (version 1.1.0) [15].

\section{Results and discussion Growth of SP-35 and removal of COD}

The strain SP-35 was able to slightly grow $\left(\mathrm{OD}_{600}\right.$ from 0.52 increased to 0.78 ) with MWL as the sole carbon source and had a faster proliferation when glucose was added as mixed carbon source; cells quickly entered into the decline phase without supplies of carbon sources (Fig. 1). These results indicate that the strain SP-35 is able to utilize MWL as the sole carbon source for cell growth to a limited extent. Moreover, strain SP-35 also utilized $\mathrm{AL}$ as a carbon source as described in previous studies [16].

The degradation rate of lignin by strain SP-35 can be determined by COD removal analysis because MWL contributes almost the entire COD loading in the culture medium when it was the sole carbon source. Our 


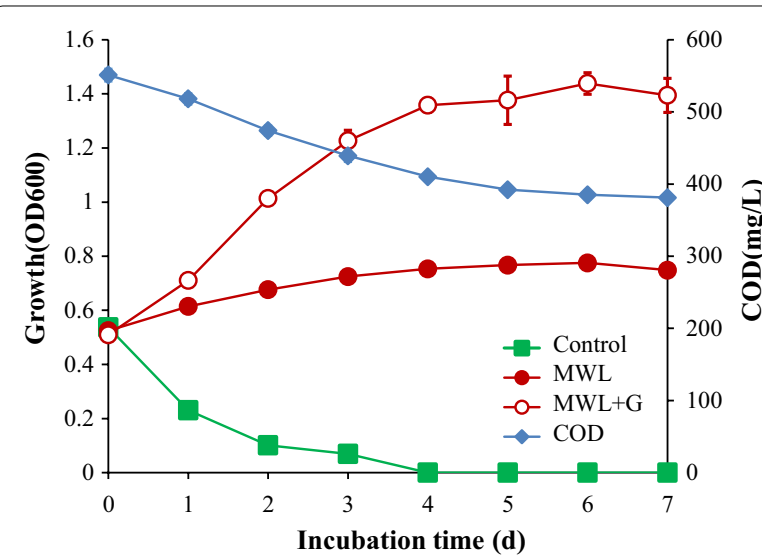

Fig. 1 Growth of the strain SP-35 during 7 days of incubation with MWL and glucose as carbon source. Symbols: closed circles, MWL as single carbon source; open circles, MWL and glucose as carbon source; closed squares, control, the growth without carbon source; closed diamond, COD with lignin as carbon source

results showed that the COD of the strain SP-35 culture was reduced from 551 to $381 \mathrm{mg} / \mathrm{L}$ after 7 days of incubation with MWL as the carbon source (Fig. 1). It was further confirmed that the MWL was degraded by the strain SP-35, yielding a 30.9\% reduction in lignin content. It is worthy to mention that previously published data reported the reduction of AL by strain SP-35 was $44.4 \%$ [16]. It is still unclear why AL is more susceptible to degradation by strain SP-35 than MWL. It should be pointed out that since the structure of MWL is closer to that of native lignin, the degradation rate of strain SP-35 to MWL represents the ability of the strain to degrade natural lignin.

To further investigate the bacterial degradation of MWL, both the un-inoculated and inoculated MWL with strain SP-35 were incubated for 7 days and were observed with SEM (Fig. 2). The size of the MWL particles treated by strain SP-35 was significantly smaller than that of the un-inoculated control samples and was reduced from 6-30 $\mu \mathrm{m}$ to $2-4 \mu \mathrm{m}$. in addition, the similar phenomenon that lignin particles become smaller also appears in other lignin degradation bacteria [4]. Meanwhile, the morphology of MWL changed from an irregular polyhedron shape to a ball shape. The variation in size and shape of the MWL particles suggest that MWL was degraded and modified.

\section{Fourier-transform infrared spectrometer spectra analysis}

The variability in the chemical composition of lignin can be identified using spectrophotometric methods because the variation in the absorbance of the individual phenolic constituents reflects the change in the chemical structures of the lignin samples. The infrared spectra
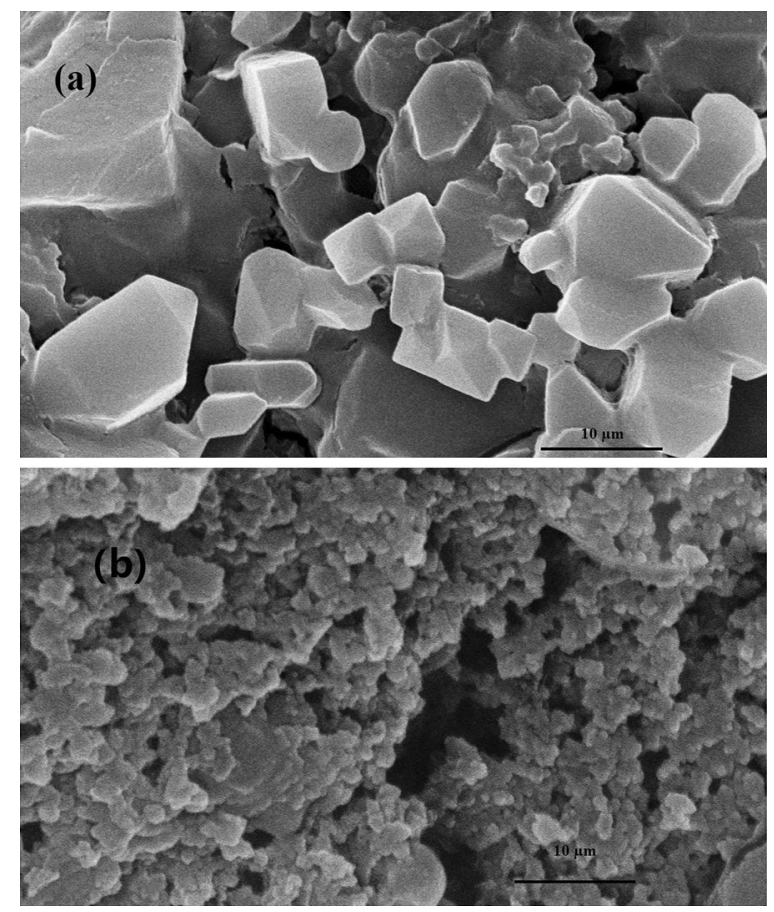

Fig. 2 Scanning electron micrograph of lignin. a MWL in un-inoculated LM medium incubated for 7 days; $\mathbf{b}$ MWL treated by 7 days of incubation LM medium with the strain SP-35

of the MWL fractions that were degraded by SP-35 for 7 days and the control are illustrated in Fig. 3. The control MWL sample showed a broad band at $3410-3460 \mathrm{~cm}^{-1}$, which originated from the hydroxyl groups in phenolic and aliphatic structures, and at $2938-2942 \mathrm{~cm}^{-1}$, which arose from $\mathrm{C}-\mathrm{H}$ stretching in aromatic methoxyl groups and in the methyl and methoxylene groups of the side chains. There are two absorption peaks around 1500 and $1630 \mathrm{~cm}^{-1}$, respectively, which originated from the skeletal and stretching vibrations of benzene rings [17]. The $\mathrm{C}-\mathrm{H}$ bending vibration in methyl groups can be assigned to the band of $1420 \mathrm{~cm}^{-1}$ and the absorbance peak at $2350 \mathrm{~cm}^{-1}$ ascribed to $\mathrm{C}=\mathrm{O}$ vibration in ketone groups. Interestingly, compared with the control sample, in the infrared spectra of MWL treated with strain SP-35 the bands from 2938 to $2942 \mathrm{~cm}^{-1}$ were weakened. FTIR results suggest that the part of $\mathrm{C}-\mathrm{C}$ and $\mathrm{C}-\mathrm{O}-\mathrm{C}$ bonds between the phenyl propane structural units of lignin may be oxidized and broken, which result in the reduced vibration of $\mathrm{C}-\mathrm{H}$ bonds and in the modification of side chains such as $\mathrm{OH}$ groups. Another interesting finding was that the absorption peak at $1630 \mathrm{~cm}^{-1}$ was weakened and the displacement of the absorption peak at $1120 \mathrm{~cm}^{-1}$ was enhanced probably due to $\mathrm{C}-\mathrm{O}$ stretch, which might be due to the production of new phenyl aromatic compounds as well as small molecules of 


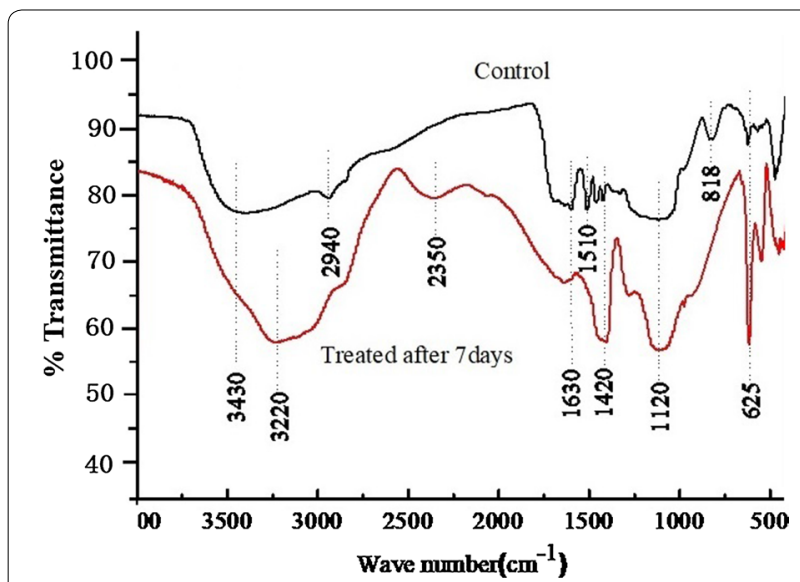

Fig. 3 FT-IR infrared spectra of the MWL fractions degraded by the strain SP-35 in LM medium after 7 days incubation

alcohols or ethers during the process of the degradation of MWL. The appearance of carbonyl group in the range of $1650-1710^{-1}$ represents $\mathrm{C}=\mathrm{O}$ bond is in conjugation with the aromatic ring, the variation suggests the production of aldehydes and acids. In addition, the vibration of the absorption peak at $818 \mathrm{~cm}^{-1}$ was weakened, proving that the side chain structure of MWL was modified. The FT-IR spectra indicated that the degradation of MWL by strain SP-35 included the oxidative cleavage of the lignin aromatic ring structures and the modification of their side chains.

\section{Aromatic intermediates identified by gas chromatography-mass spectrometry analysis}

To determine the intermediate metabolites, cells of SP-35 were incubated in LM medium with MWL or AL as the sole carbon source for 7 days and the fractions were collected and detected with GC-MS from cultures in the first, third, fifth and seventh day. Hence, a total of 20 single-ring aromatic compounds were identified in both the MWL and AL lignin cultures (Table 1). As can be seen from parts A and B of Fig. 4, most of the absorption peak areas of the aromatic compounds identified from lignin cultures increased during the 7-day incubation and reached its highest point during the 5th and 7th days in the MWL and AL cultures, respectively. Notably, the peak area of certain aromatic compounds significantly increased compared with the control, such as that of ferulic acid (compound 20), which increased 29.3 times, the peak of 4-hydroxycinnamic acid (HCA, compound 18 , which increased 8.3 times, and the peak of 2,4-dihydroxybenzaldehyde (2,4-DHB, compound 5), which increased 6.4 times in MWL cultures (Fig. 4a). In addition, the peak area of guaiacol (compound 1), 4'-hydroxyacetophenone (HAP, compound 12), vanillin (compound 10) and 3,4-dihydroxyphenylglycol (DHPG, compound

Table 1 Aromatic metabolites of lignin degradation by strain SP-35 lignin

\begin{tabular}{|c|c|c|c|c|c|c|}
\hline No. & Retention time & & MWL & MWLC & $A L$ & ALC \\
\hline 1 & 13.644 & Guaiacol & + & - & + & - \\
\hline 2 & 14.208 & 3-Methylphenol & + & + & + & + \\
\hline 3 & 14.82 & Phenylacetic acid & - & - & + & + \\
\hline 4 & 16.192 & 4-Hydroxybenzaldehyde & - & - & - & + \\
\hline 5 & 16.23 & 2,4-Dihydroxybenzaldehyde & + & + & - & - \\
\hline 6 & 16.286 & 3,4-Dihydroxyphenylglycol & + & - & + & + \\
\hline 7 & 17.622 & Butylated hydroxytoluene & + & + & + & + \\
\hline 8 & 17.979 & Methyl 2,5-dihydroxybenzoate & + & - & - & - \\
\hline 9 & 18.496 & 4-Hydroxybenzoate & + & - & + & - \\
\hline 10 & 18.562 & Vanillin & + & + & + & + \\
\hline 11 & 18.872 & Acetovanillone & - & - & - & + \\
\hline 12 & 19.549 & 4'-Hydroxyacetophenone & + & - & + & + \\
\hline 13 & 20.339 & Vanillic acid & + & - & + & - \\
\hline 14 & 20.678 & 3,4-Dihydroxymandelic acid & - & - & + & - \\
\hline 15 & 20.734 & Syringaldehyde & + & - & - & - \\
\hline 16 & 21.797 & 4-(3-hydroxybutyl)-2-methoxyphenol & - & - & + & - \\
\hline 17 & 21.806 & Diydroferulic acid & + & - & - & - \\
\hline 18 & 22.173 & 4-Hydroxycinnamic acid & + & + & + & - \\
\hline 19 & 23.452 & Butyl 2-ethylhexyl phthalate & - & - & + & - \\
\hline 20 & 23.856 & Ferulic acid & + & + & + & - \\
\hline
\end{tabular}



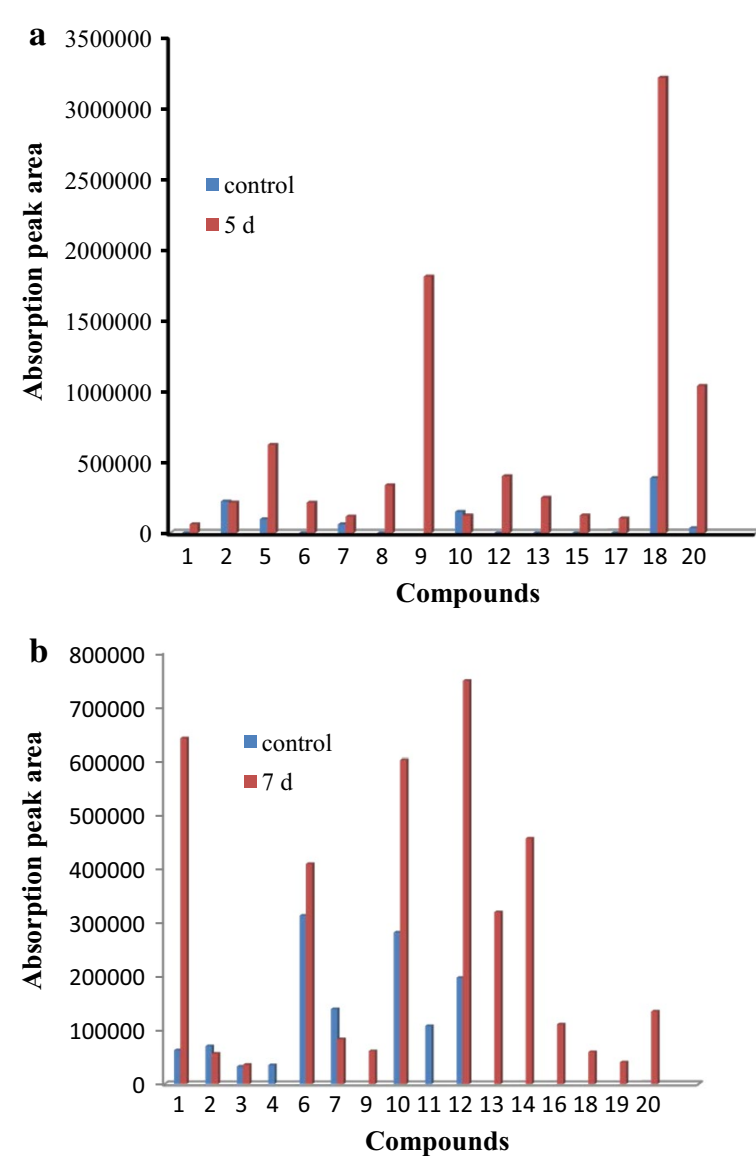

Fig. 4 Comparison of peaks of aromatic compounds identified by GC-MS in the first and fifth days of incubation of MWL (a) and at first and seventh days of incubation of AL (b). Numbers representing the aromatic compounds are shown in Table 1

6) increased 10.2, 3.8, 2.1 and 1.3 times in AL cultures, respectively (Fig. $4 \mathrm{~b}$ ). Therefore, the increased proportion in the peaks of aromatic compounds in the fifth day of incubation of MWL indicated that HCA was the most abundant aromatic metabolite and constituted 37\% of aromatic metabolites. There were other major aromatic compounds, including 4-hydroxybenzoic acid (HBA, compound 9, 21\%), ferulic acid (12\%), 2,4-DHB (7\%), and HAP (5\%); these aromatic compounds, together with HCA, accounted for $82 \%$ of all aromatic metabolites (Fig. 5a). Unlike in MWL, the predominant aromatic compounds in AL culture included HAP (20\%), guaiacol (17\%), vanillin (16\%), 3,4-dihydroxymandelic acid (DOMA, compound 14, 12\%), DHPG (11\%), and vanillic acid (compound 13, 8\%), which together constituted 83\% of all aromatic metabolites (Fig. 5b). However, previous studies have shown that the major degradation products of AL when degraded by the strain Bacillus ligniniphilus L1 included vanillic acid (44.2\%), HAP (14.5\%), vanillin
(8.7\%), and 4-hydroxyphenylacetic acid (7.2\%) [4]. Based on these results, we suspected that strain SP-35 and strain L1 have different metabolic pathways for lignin degradation and the production of their respective intermediates. To further explore this claim, metabolic pathway analysis based on whole-genome sequencing should be performed. It must be pointed out that the uncultured MWL also contained small amounts of monomers such as 3-methylphenol (3-MP, compound 2), 2,4-DHB, butylated hydroxytoluene (BHT, compound 7), vanillin, HCA and ferulic acid. The reason why uncultured MWL also contained small amounts of monomers, probably is due to the cleavage of $\beta-O-4$ linkages and ester bonds (acetyl and coumaryl residues) caused by condensation and oxidation of side chains during the preparation of MWL., Although the single ring compounds are produced during the preparation of MWL, but wouldn't significantly change the core of the lignin structure [18]. In addition, the tolerance of strain SP-35 to the aromatic compounds was tested with more than 20 aromatic compounds, the results indicate that when the concentration of the compound was $0.1 \%$, strain SP-35 was able to grow under the presence of most of the tested compounds, respectively. When the compound concentration was increased to $0.5 \%$, the growth of cells was observed only with 14 compounds as substrates, and when the compound concentration was increased to $5 \%$, the cells could not grow in the presence of any compound (Additional file 1). Our findings suggest that strain SP-35 could be used as a potential cell factory for the production of high-value aromatic compounds with lignin that might be widely used in the pharmaceutical, fine chemicals, and food industries.

\section{Lignin degradation and its intermediates metabolic pathways analysis based on the whole-genome sequencing and annotation}

With the aim of getting a better understanding of the mechanisms of lignin degradation by strain SP-35 and exploring the catabolic pathways of intermediate metabolites, the whole genome sequence of strain SP35 was obtained by the SMRT method, using the PacBio RS II platform (Whole Genome Shotgun project has been deposited at DDBJ/EMBL/GenBank under the accession no. CP021455.1). Based on the annotation analysis (Additional file 2) of the genome of the strain SP-35, a total of 94 genes that may be involved in the degradation and metabolism of lignin were identified (Table 2). These genes belong to 34 enzymes, one transcriptional regulator, one degradation regulator and three transporters, respectively. It is notable that the genome of the strain SP-35 contained a DyPs and one Lacc encoding genes that is probably involved in the oxidation of 

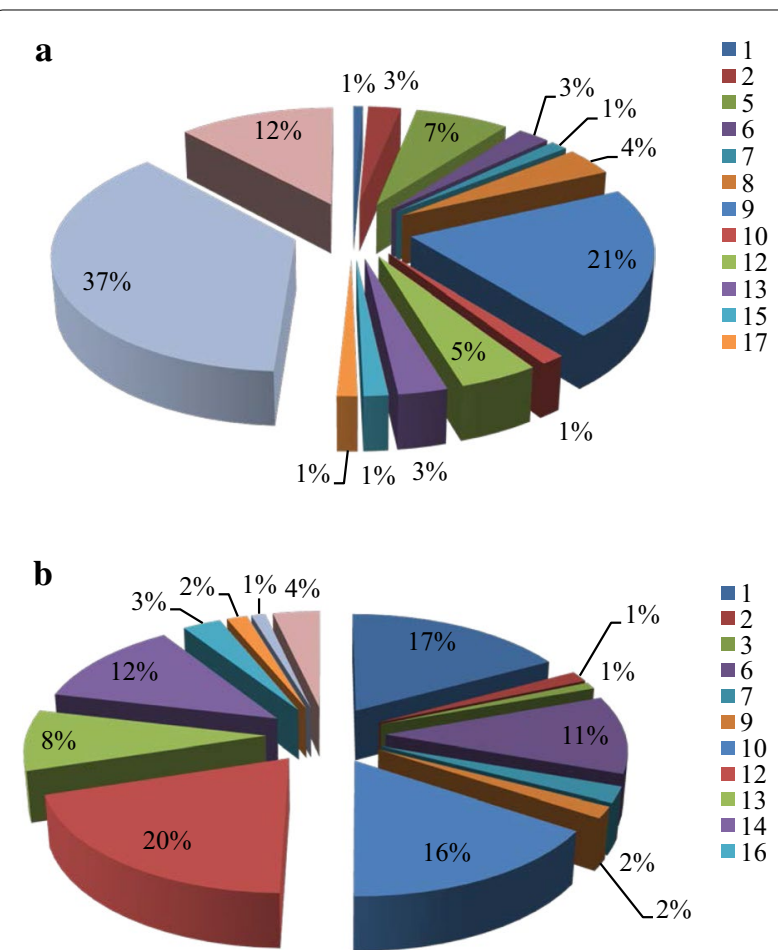

Fig. 5 Proportion of peaks areas of aromatic compounds in the fifth day of incubation of MWL (a) and seventh day of incubation of AL (b). Numbers representing the aromatic compounds are shown in Table 1

lignin, but no LiP or MnP genes were observed. It is supported by published data which showed that the maximum crude enzyme activity of DyPs reached $648.4 \mathrm{u} / \mathrm{L}$ while Lacc only reached $70.1 \mathrm{u} / \mathrm{L}$ in the fourth day of the culturing of strain SP-35 [16]. The gene sequence identities of encoding DyPs of strain SP-35 with that of Pseudomonas (CP015878.1) and Rhodococcus (FN563149.1) are $73-69 \%$, however, there are no significant similarity found with that of fungi (Polyporaceae sp. U77073.1). According to the sequence information of Genbank, the fungal and bacterial DyPs show hardly sequence homology and belong to different protein families and subfamilies $[19,20]$. In addition, there were four alkylhydroperoxidase, one glutathione peroxidase, and three superoxide dismutase encoding genes observed in the genome of strain SP-35, which might also be involved in the degradation of lignin. Therefore, the DyPs of strain SP-35 may have a potentially great application in lignin degradation, dye discoloration, wastewater treatment, etc.

The $\beta$-aryl ether linkage is the most abundant linkage in lignin. It comprises $45-50 \%$ of all the intermonomer linkages in softwood and $60-62 \%$ of all the intermonomer linkages in hardwood lignin [9]. Genome annotation revealed the presence of sixteen genes of glutathione transferases (GSTs) and two genes of NAD-dependent alcohol dehydrogenase (NAD-ADH) in the genome of strain SP-35, which may be involved in the cleavage of the $\beta$-aryl ether linkage, other than that, these genes are also very important for normal celluar process (Table 2). The $\beta$-aryl ether cleavage pathway was uncovered in strain Spihingobuim sp. SYK-6 by the degradation of guaiacylglycerol- $\beta$-guaiacyl ether (GGE). [6]. We speculate that in strain SP-35, two NAD-ADH molecules are probably involved in the conversion of GGE to $\alpha$-(2methoxyphenoxy)- $\beta$-hydroxypropiovanillone (MPHPV), some GSTs genes with $\beta$-etherase activity may participate in the degradation of MPHPV to generate $\beta$-glutathionyl$\gamma$-hydroxypropiovanillone (GS-HPV), and some GSTs genes with the activity of GSH-dependent lyase are able to catalyze GS-HPV to produce glutathione disulfide (GSSG) and $\gamma$-hydroxypropiovanillone (HPV). The specific substrates and activities of these enzymes and whether they participate in the $\beta$-aryl ether cleavage pathway need further validation in the next step work of our investigation. In addition, MPHPV, HPV and vanilloyl acetic acid (VVA) were identified in the third and fifth days of strain SP-35 culture (MM63 medium) with $100 \mathrm{mg} / \mathrm{L}$ GGE as solo carbon source incubated 7 days at $30{ }^{\circ} \mathrm{C}$ (Fig. 6). In the $\beta$-aryl ether cleavage pathway of SYK-6, VVA was obtained from the conversion of HPV [7]. These data further confirmed the presence of the $\beta$-aryl ether cleavage pathway in strain SP-35. So far, there are few information available about the $\beta$-aryl ether catabolic system except for the three strains of Sphingomonadaceae, and it is believed that the $\beta$-aryl ether catalyzed by GSTs as a lignin degradation strategy may exist only in Sphingomonadaceae [7]. Based on the comparison of the homology of the enzyme-encoding gene, the previous articles concluded that the $\beta$-aryl ether metabolic pathway may exist only in the Sphingomonadaceae family. However, our results indicate that other species in bacteria kingdom may also have a $\beta$-aryl ether cleavage pathway, but the homology of the coding gene of enzymes involving the pathway compare to Sphingomonas is too low to be confirmed based on homology comparison. Thus, the elucidation of the beta-aryl ether catabolic system of strain SP-35 will update the understanding of the role of the beta-aryl ether cleavage pathway in lignin-degrading bacteria. Based on the genome annotations and GC-MS data, we can predict the metabolic pathway of the aromatic intermediate metabolites of strain SP-35 (Fig. 7 and 8). According to the unit type of lignin, the pathways were divided into three categories, including $\mathrm{H}, \mathrm{G}$ and $\mathrm{S}$ types. Among the three categories, the $\mathrm{H}$ type has multiple pathways, including benzoate, 3-MP, phenylacetic acid (PA, compound 3), 4-hydroxybenzaldehyde (HBAld, compound 4), HAP, HCA, HAP 
Table 2 Putative enzyme's genes of strain SP-35 involving in lignin degradation and catabolic

\begin{tabular}{|c|c|c|c|}
\hline No & Encode protein & Orf-name & KO/Gene_ID \\
\hline 1 & Dyp-type peroxidase & orf02723_1 & K07223 \\
\hline 2 & Alkylhydroperoxidase & orf01844_1, orf01464_1, orf00109_1, orf01615_1 & \\
\hline 3 & Glutathione peroxidase & orf01521_1 & K00432 \\
\hline 4 & Laccase & orf02918_1 & K05810 \\
\hline 5 & Cytochrome P450 & orf00617_1, orf01862_1, orf00094_1 & \\
\hline 6 & Superoxide dismutase & orf00200_1, orf01398_1, orf02451_1 & $\begin{array}{l}\text { K04565, } \\
\text { K04564 }\end{array}$ \\
\hline 7 & Benzoate 1,2-dioxygenase & orf01595_1, orf01596_1 & $\begin{array}{l}\text { K05549, } \\
\text { K05550 }\end{array}$ \\
\hline 8 & 1,6-Dihydroxycyclohexa-2,4-diene-1-carboxylate dehydrogenase & orf01598_1 & K05783 \\
\hline 9 & Catechol 1,2-dioxygenase (CatA) & orf01594_1 & K03381 \\
\hline 10 & Muconate cycloisomerase (CatB) & orf01593_1 & K01856 \\
\hline 12 & $2,4^{\prime}$-Dihydroxyacetophenone dioxygenase & orf00887_1 & \\
\hline 13 & 4-Hydroxybenzoate polyprenyltransferase & orf04164_1 & K03179 \\
\hline 14 & Phenylacetate-CoA ligase (paaK) & orf02154_1 & K01912 \\
\hline 15 & 1,2-Epoxyphenylacetyl-CoA isomerase (paaG) & $\begin{array}{l}\text { orf00429_1, orf01010_1, orf00301_1, orf01175_1, } \\
\text { orf03337_1 }\end{array}$ & K15866 \\
\hline 16 & 3-Oxoadipyl-CoA thiolase (paaJ) & orf01279_1 & \\
\hline 17 & 3-Hydroxyadipyl-CoA dehydrogenase (paaH) & orf01817_1 & \\
\hline 18 & 4-Coumarate-CoA ligase & orf00090_1, orf00074_1, orf04303_1,orf00300_1 & K00666 \\
\hline 19 & p-Hydroxyphenylacetate 3-hydroxylase & orf00203_1 & \\
\hline 20 & R)-Benzylsuccinyl-CoA dehydrogenase & orf03060_1 & K00249 \\
\hline 21 & Hydroxycinnamoyl-CoA hydratase-lyase & orf03793_1 & K15866 \\
\hline 22 & Vanillate O-demethylase oxidoreductase & orf00093_1, orf01861_1 & \\
\hline 23 & 4-Hydroxybenzoyl-CoA thioesterase & orf02427_1, orf03445_1 & K07107 \\
\hline 24 & Aldehyde dehydrogenase & orf01894_1, orf02026_1, orf01760_1, orf00092_1 & K00128 \\
\hline 25 & Alcohol dehydrogenase & orf02362_1, orf01315_1, orf00315_1 & \\
\hline 26 & p-Hydroxybenzoate hydroxylase/Baeyer-Villiger monooxygenase & orf01314_1 & \\
\hline 27 & Muconolactone D-isomerase (CatC) & orf01601_1 & K03464 \\
\hline 28 & 3-Oxoadipate CoA-transferase subunit A (pcal) & orf01277_1 & K01031 \\
\hline 29 & 3-Oxoadipate CoA-transferase subunit B (pcaJ) & orf01278_1 & K01032 \\
\hline 30 & Carboxymuconolactone decarboxylase (pcaC) & orf00668_1, orf02483_1 & K01607 \\
\hline 31 & Beta-ketoadipate enol-lactone hydrolase (pcaD) & orf01600_1, orf01280_1 & K01055 \\
\hline 32 & Beta-ketoadipyl CoA thiolase (pcaF) & orf02814_1, orf01237_1, orf01537_1, orf02199_1 & \\
\hline 33 & Glutathione S-transferase & $\begin{array}{l}\text { orf00149_1, orf03152_1, orf01651_1, orf01341_1, } \\
\text { orf03018_1, orf01342_1, orf02126_1, orf02328_1, } \\
\text { orf01306_1, orf00150_1, orf00388_1, orf02845_1, } \\
\text { orf00142_1, orf03308_1,orf03594_1, orf01202_1 }\end{array}$ & \\
\hline 34 & NAD-dependent alcohol dehydrogenase & orf01643_1, orf03396_1 & \\
\hline 35 & Beta-ketoadipate pathway transcription regulator & orf01276_1, orf00869_1 & \\
\hline 36 & Gentisate transporter & orf03503_1 & K05548 \\
\hline 37 & Benzoate transporter & $\begin{array}{l}\text { orf00892_1, orf01603_1, orf00360_1, orf03205_1, } \\
\text { orf01599_1 }\end{array}$ & K05782 \\
\hline 38 & Alpha-ketoglutarate transporter (benE) & orf01819_1 & K03761 \\
\hline 39 & Benzoate anaerobic degradation regulator & orf04233_1 & \\
\hline
\end{tabular}

and DHPG degradation pathways, and both the $\mathrm{G}$ and $\mathrm{S}$ types have only one pathway, namely the ferulic acid degradation and syringaldehyde (SA, compound 15) degradation pathways, respectively.
The $\beta$-KAP is a chromosomally encoded convergent pathway for the degradation of aromatic compounds derived from plants, including lignin, as the primary source of carbon and energy and is widely distributed 


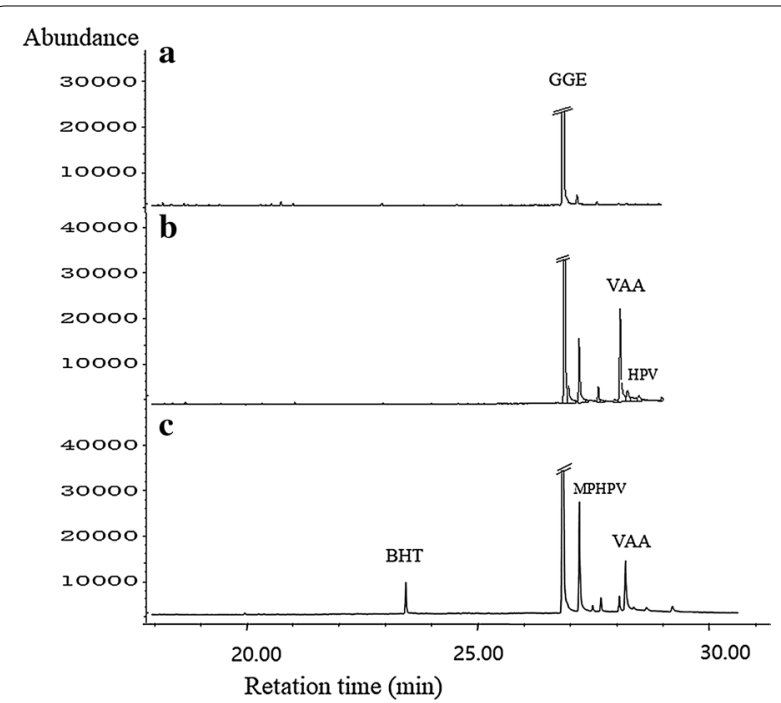

Fig. 6 GC-MS spectra of culture of strain SP-35 with GGE as single carbon source. a Un-inoculated culture (control); $\mathbf{b}$ the third day of incubation; $\mathbf{c}$ the fifth day of incubation. GGE guaiacylglycerol- $\beta$-guaiacyl ether; MPHPV: $\alpha$-(2-methoxyphenoxy)- $\beta$ hydroxypropiovanillone, GS-HPV $\beta$-glutathionyl- - -hydroxypropiovanill one, HPV $\gamma$-hydroxypropiovanillone, WA: vanilloyl acetic acid

in soil bacteria and fungi $[8,21]$. As we all know, the $\beta$-KAP includes two branches of catechol and protocatechuate, and both branches observed should be present in strain SP-35 (Fig. 8). All the enzyme encoding genes involved in the catechol branch have been identified in the genome of strain SP-35, including catechol 1, 2-dioxygenase (cat $A)$, muconate cycloisomerase (catB), muconolactone $\mathrm{D}$-isomerase (catC), $\beta$-ketoadipate enollactone hydrolase ( $p c a D)$, 3-oxoadipate CoA-transferase subunit A (pcaI), 3-oxoadipate CoA-transferase subunit $\mathrm{B}(p c a)$ ), and $\beta$-ketoadipyl CoA thiolase $(p c a F)$. In addition, the genes corresponding to the enzymes involved in the PA branch, such as the carboxymuconolactone decarboxylase $(p c a C)$ gene, have been observed, but no genes encoding the protocatechuate 3, 4-dioxygenase (pcaHG) and $\beta$-carboxymuconate cycloisomerase ( $p c a B)$ were identified. However, protocatechuate was degraded and was used as the sole carbon source by strain SP-35, proving the presence of the protocatechuate degradation pathway (Table 3). It may be due to the homology of pcaHG and pcaB in strain SP-35 compared with other bacteria was too low to be identified based on sequence alignment, and this hypothesis needs further study to confirm. In addition, a $\beta$-KAP transcription regulator (pcaR) encoding gene was identified in the genome of strain SP-35 and was required for both the induction of the pca regulon (pcaBDC, pcaIJ, and pcaF) and the chemotactic response of the bacteria to aromatic compounds [22]. It provided additional evidence for the presence of the protocatechuate branch in strain SP-35. In addition, the protocatechuate branch of the KAP pathway of SP-35 differs from the fungus in that 4-carboxymuconolactone is converted to beta-carboxymuconolactone by $p c a B$ and then further converted to 3-oxoadipate instead of being converted to beta-ketoadipate-enol-lactone via $p c a C[8]$.

Benzoate has been widely used as a model compound to study the catabolism of aromatic compounds in bacteria [23]. In strain SP-35, the catabolic pathway of benzoate is as follows: First, benzoate is converted to cis-1, 2-dihydroxycyclohexa-3, 5-diene-1-carboxylate (DHCDC) by the catalysis of benzoate 1, 2-dioxygenase, and is then converted to catechol by DHCDC dehydrogenase. After that, the benzene ring of catechol is cleaved by cat A via a ring opening reaction and converted to cis, cis-muconate, where the metabolic pathway then leads into the $\beta$-KAP. The benzoic acid degradation pathway of strain SP-35 is completely different from that of fungi, which is usually converted to p-hydroxybenzoic acid via benozate 4-monooxygenase and then converted to protocatechuic acid, such as in Rhodotorula graminis and Aspergillus niger [24]. Interestingly, there are three benE and two benK genes encoding proteins belonging to the benzoate transporter super family. It was proposed that the products of the benE and benK genes function as transporters, taking up benzoate into cells to promote the degradation of benzoate [25]. Another interesting finding was that there is a benzoate anaerobic degradation regulator encoding gene ( $\mathrm{BadR})$ that is able to improve the anaerobic degradation of benzoate [26]. It was suggested that besides the above mentioned benzoate degradation pathway, there may be other anaerobic degradation pathways in strain SP-35. However, strain SP-35 as an aerobic bacterium cannot grow under anerobic conditions using benzoic acid as a single carbon source. Furthermore, the encoding genes of enzymes involving in anaerobic degradation of benzoate such as benzoate-CoA ligase, benzoylCoA oxygenase and reductase, benzoyl-CoA hydratease and 3,4-dehydroadipyl-CoA semialdehyde dehydrogenase have not been found in the genome of strain SP-35 $[27,28]$. Therefore, the strain SP-35 does not have an anaerobic degradation pathway of benzoate.

$3-\mathrm{MP}$ is a precursor to numerous compounds and its metabolic pathway in strain SP-35 was predicted as below. First, 3-MP was converted to 4-methylcatechol via phenol 2-monooxygenase, and then 4-methylcatechol was catalyzed into 3 -methyl-cis, cis-muconate by the ring-opening reaction of catA. Previous research showed that catA not only uses catechol as substrate, but also is able to catalyze the methyl-substituted catechols, such as 3-methylcatechol and 4-methylcatechol [29]. However, the phenol 2-monooxygenase gene has not been identified in the genome of strain SP-35 yet and further effort is 


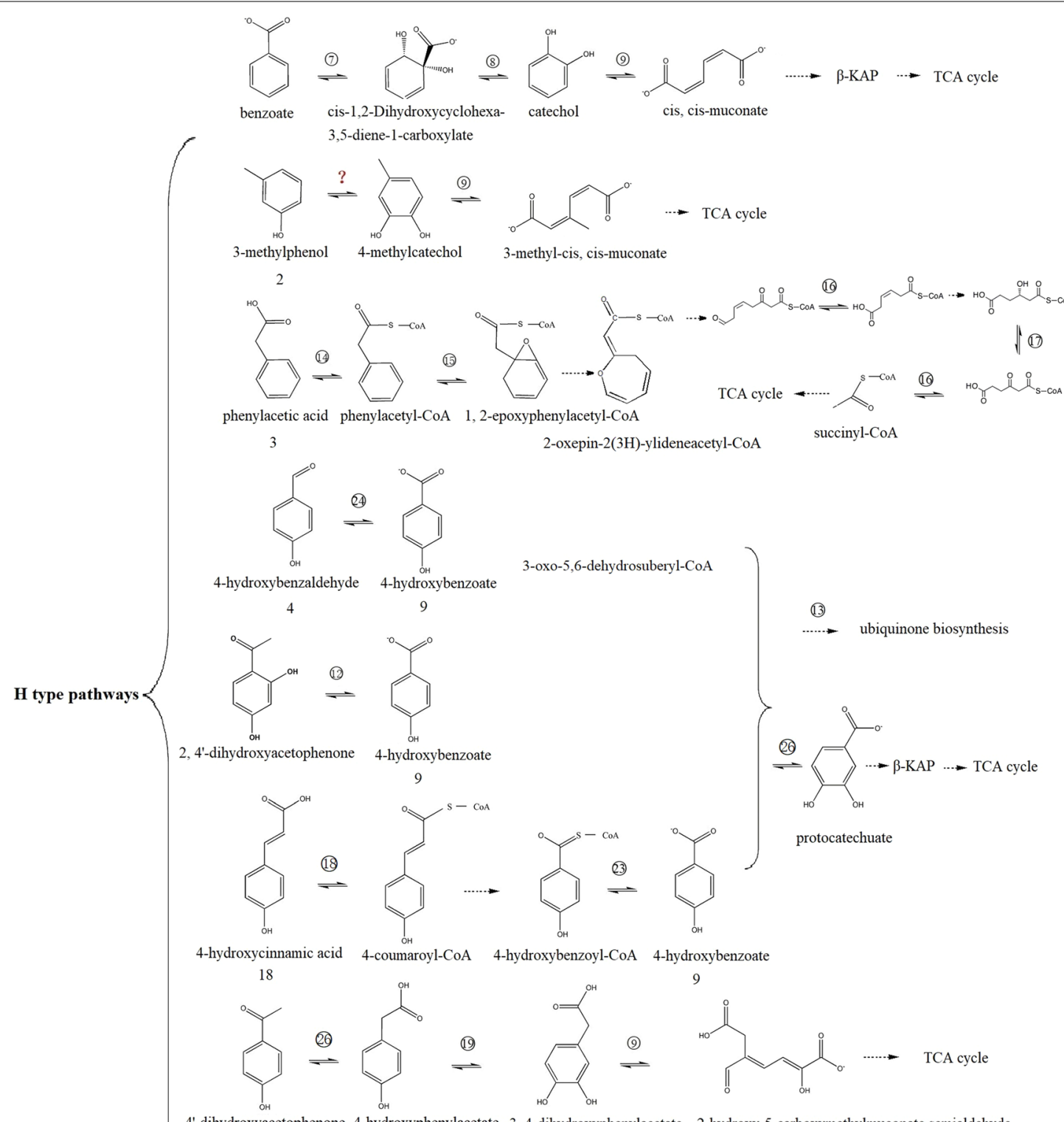

4'-dihydroxyacetophenone 4-hydroxyphenylacetate 3,4-dihydroxyphenylacetate 2-hydroxy-5-carboxymethylmuconate semialdehyde

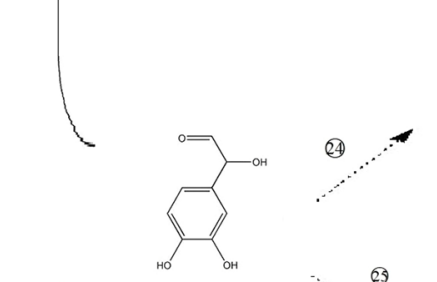

3,4-dihydroxyphenylglycolaldehyde

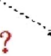

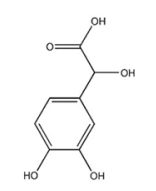

3,4-dihydroxymandelic acid

14

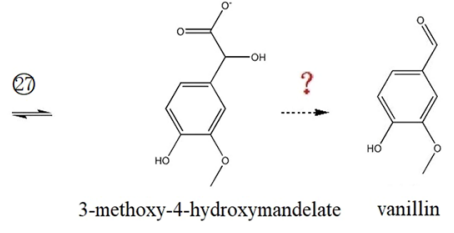

(24) 1

10
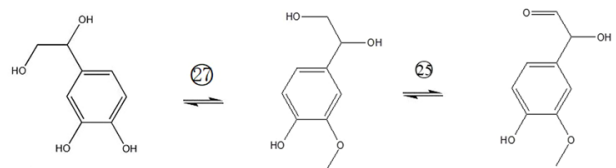

34-dihydroxyphenylglycol 3-methoxy-4-hydroxyphenylglycol 3-methoxy-4-hydroxyphenylglycolaldehyde

Fig. 7 Putative catabolic pathway of lignin derived monomers and catabolism genes in Comamonas serinivorans SP-35. H type pathway. Symbol "?" means the enzyme encoding gene was un-confirmed. The reactions indicated by dashed arrows have not been confirmed. Abbreviations: Numbers represent the aromatic compounds were shown in Table 1, and the circled numbers representing the number of enzymes are shown in Table 2 

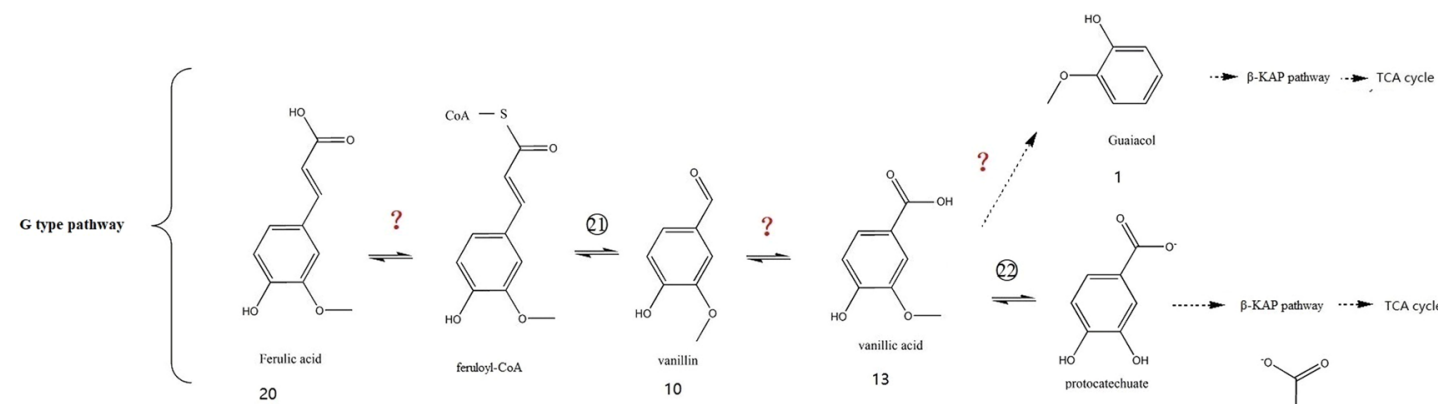<smiles>COc1cc(C(=O)O)cc(OC)c1OCCO</smiles><smiles>COc1cc(C(=O)O)cc(OC)c1O</smiles>
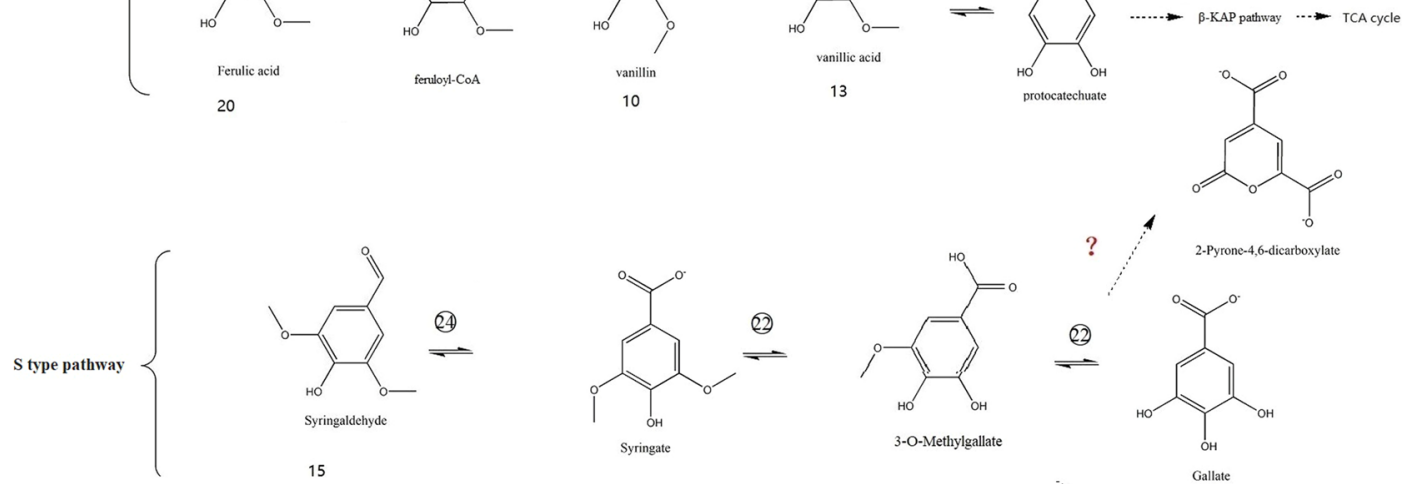

(4)

3-O-Methylgaallate
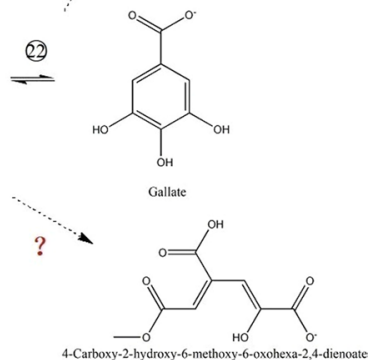

B-KAP pathway \{
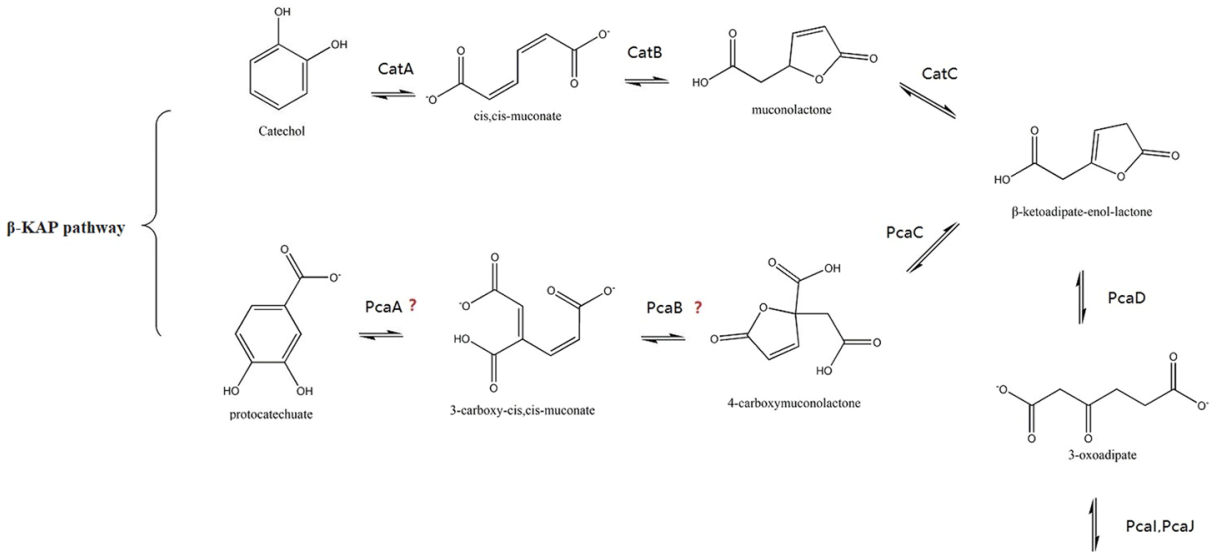

Pcac
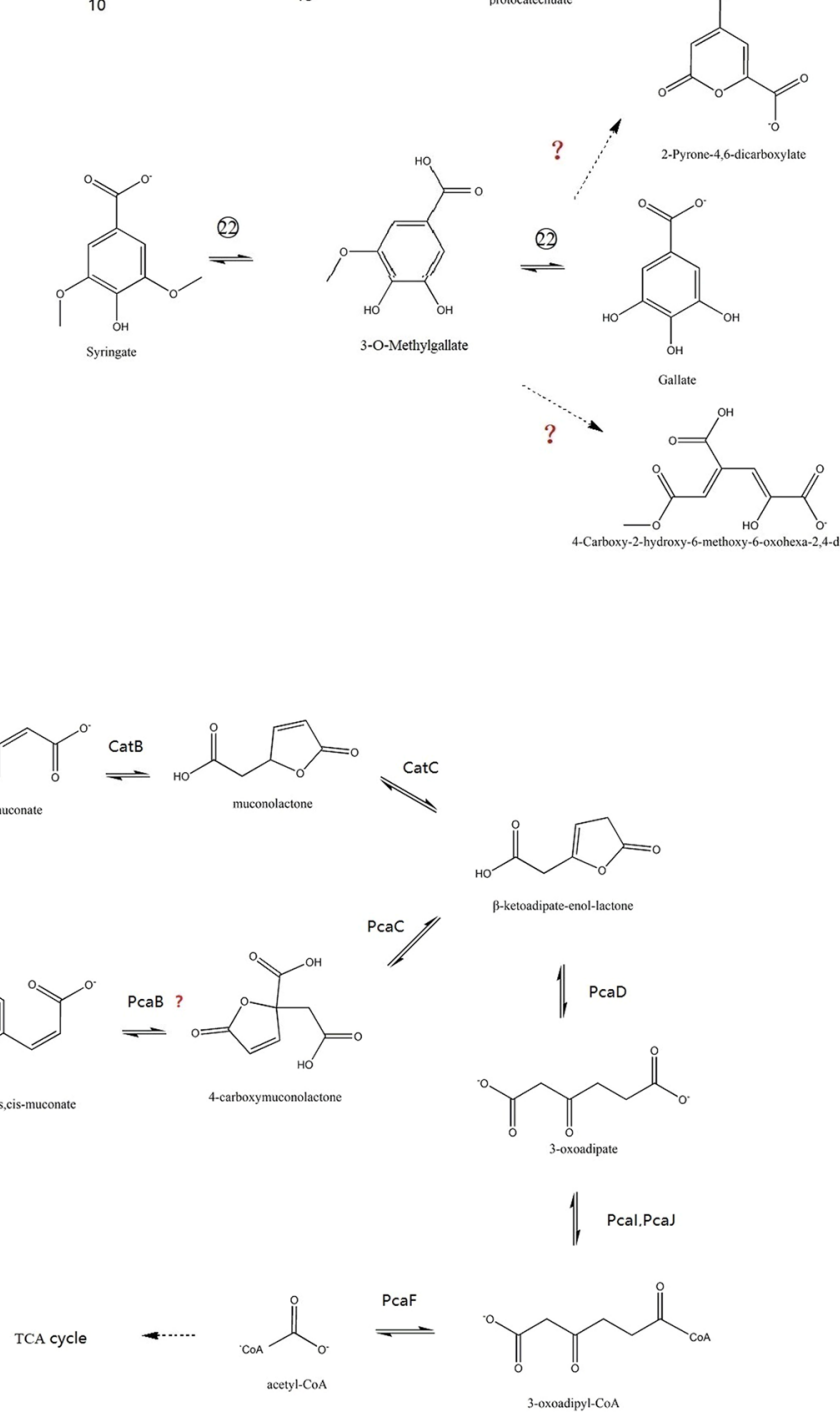

Fig. 8 Putative catabolic pathway of lignin derived monomers and catabolism genes in Comamonas serinivorans SP-35. G and S pathway and B-KAP. Symbol "?" means the enzyme encoding gene was un-confirmed. The reactions indicated by dashed arrows have not been confirmed. Abbreviations: Numbers represent the aromatic compounds were shown in Table 1, and the circled numbers representing the number of enzymes are shown in Table 2

required to obtain the encode gene of phenol 2-monooxygenase in the future.

Contrary to benzoate and 3-MP, the metabolic pathway of PA was not via $\beta$-KAP in the strain SP-35. PA was reduced to phenylacetyl-CoA (PA-CoA) by the reduction catalytic of PA-CoA ligase (paaK), and PA-CoA was converted to 1, 2-epoxyphenylacetyl-CoA (ep-CoA) by ring 1,2- phenylacetyl-CoA epoxidase (paaABCDE), 
Table 3 The degradation of aromatic monomers by the strain SP-35

\begin{tabular}{lc}
\hline Compounds & $\begin{array}{l}\text { Completely } \\
\text { degraded time } \\
\text { (hours) }\end{array}$ \\
\hline Ferulic acid & 48 \\
4-Hydroxybenzoic acid & 24 \\
4-Coumaric acid & No \\
Gentisate & 24 \\
Protocatechuate & 24 \\
Benzoate & 168 \\
Vanillin & 72 \\
Vanillic acid & 36 \\
\hline
\end{tabular}

then ep-CoA was converted to 2-oxepin-2(3H)-ylideneacetyl-CoA by ring 1,2-epoxyphenylacetyl-CoA isomerase (paaG). 2-oxepin-2(3H)-ylideneacetyl-CoA was subsequently converted into 3-oxo-5,6-dehydrosuberylCoA, which was catalyzed by oxepin-CoA hydrolase (paaZ). 3-oxo-5,6-dehydrosuberyl-CoA was converted to 2,3-dehydroadipyl-CoA via the catalyzation of 3-oxoadipyl-CoA thiolase (paaJ), then 2,3-dehydroadipyl-CoA was hydrolyzed into 3-hydroxyadipyl-CoA by 2,3-dehydroadipyl-CoA hydratase ( $p a a F)$. The latter intermediate was converted to 3-oxoadipyl-CoA by 3-hydroxyadipylCoA dehydrogenase (paaH) and finally biotransformed to succinyl-CoA by paaJ. There is one paaK, and five paaG, one paaJ and one paaH encoding genes that were identified in the genome of strain SP-35, which may be involved in the PA degradation pathway. The PA utilization pathway represents one of the strategies for the degradation of a variety of aromatic compounds in bacteria and is different with another strategy which employs two multicomponent monooxygenases or a dioxygenase convert aromatic compounds to catechol and subsequently cleaving the bond and into $\beta$-KAP pathway [30]. In addition, unlike the bacterial degradation PA pathway of strain SP-35, the aerobic metabolism of PA by fungi usually catalyzed by monooxygenases and generated homogentisate [31].

As the second major metabolite of MWL, HBA probably was biotransformed from three intermediate metabolites in strain SP-35, including HCA, HBAld and 2, 4'-dihydroxyacetophenone (DHAP). We gathered the pathway from HCA to HBA as follows first, 4-coumarate-CoA ligase catalyzes the ligation of CoA to $\mathrm{HCA}$ and produces 4-coumaroyl-CoA, then converts it to 4-hydroxybenzoyl-CoA (HBA-CoA) by beta-oxidation reaction, the beta-oxidation was a multi-step reaction and the enzymes involved in the reaction are still not clear [32], Finally, HBA-CoA was further converted to $\mathrm{HBA}$ via $\mathrm{HBA}-\mathrm{CoA}$ thioesterase. In addition, the HBA was also yielded by the catalysis of HBAld by aldehyde dehydrogenase (ALDH) or from DHAP by DHAP dioxygenase. It is worthwhile mentioning that although most dioxygenases cleave within the aromatic ring of the substrate, DHAP dioxygenase is very unusual in that it is involved in the aliphatic $\mathrm{C}-\mathrm{C}$ bond cleavage in a substituent of the aromatic ring [33]. The fate of HBA includes two branches, one of which has been converted to 4-hydroxy-3-polyprenylbenzoate by HBA polyprenyltransferase and into ubiquinone biosynthesis pathway via multistep reaction. Another branch of the metabolic pathway is the conversion of HBA to protocatechuate by $p$-hydroxybenzoate hydroxylase, which is then metabolized via the $\beta$-KAP.

HAP is the main metabolite of lignin with the highest content in AL culture and its hypothesized metabolic pathway, including a 3-step reaction, is described. The first step is the conversion of HAP to 4-hydroxyphenylacetate (HPA) by the catalysis of 4-hydroxyacetophenone monooxygenase (HAPMO), and then, the catalysis of HPA by $p$-hydroxyphenylacetate 3-hydroxylase, producing 3,4-dihydroxyphenylacetate (HPCA), followed by the ring-opening reaction of catA to convert HPCA to 2-hydroxy-5-carboxymethylmuconate semialdehyde. We found an enzyme encoding gene for Baeyer-Villiger monooxygenase in the genome of SP-35 which might have HAPMO activity. A HAPMO, purified from Pseudomonas fluorescens and showing the characteristics of a Baeyer-Villiger-type monooxygenase was reported in the past [34].

The metabolite DHPG has strong antioxidant activity and radical scavenging properties and was found to originate from plants such as the olive fruit as well as medicinal plants [35]. So far there are no published reports on the bacterial depolymerization of lignin to produce DHPG and no information on the bacterial metabolic pathway of DHPG. However, DHPG is an important metabolite in mammalian metabolism, and several metabolic pathways of DHPG were identified in mammalian [36]. Therefore, based on the metabolic pathways of mammals, we surmised the metabolic pathways of DHPG and DOMA as follows. First, the 3,4-dihydroxyphenylglycolaldehyde is converted to DHPG or DOMA by the catalysis of alcohol dehydrogenase $(\mathrm{ADH})$ or $\mathrm{ALDH}$, respectively. Then the DHPG is converted to 3-methoxy-4-hydroxyphenylglycol (MHPG) via catechol $O$-methyltransferase (COMT). After that, MHPG is converted to 3-methoxy-4-hydroxyphenylglycolaldehyde (MOPEGAL) by $\mathrm{ADH}$ and is then biotransformed to 3-methoxy-4-hydroxymandelate (VMA) by ALDH. DOMA was also converted to VMA via COMT. The COMT has already 
been identified in bacteria, which have similar substrates with those of mammals [37]. Even though we have found six ALDH and nine ADH encoding genes in the genome of strain SP-35, their catalytic function in the metabolic pathways of DHPG and DOMA remains to be further confirmed.

Ferulic acid is an extremely abundant phenolic cinnamic acid derivative in the plant kingdom, and ferulic acid and $\rho$-coumaric acids are predominantly linked at the benzyl position of lignin. As shown in Fig. 8, the hypothetical degradation pathway of ferulic acid in the strain SP-35 is discussed below. Ferulic acid is biotransformed into vanillic acid with feruloyl-CoA and vanillin as intermediates, by the catalytic functions of feruloylCoA synthetase and vanillin dehydrogenase, respectively. The metabolism of vanillic acid is described in the conversion of $\mathrm{O}$-demethylated to protocatechuate. Another possible pathway for the degradation of vanillic acid is the conversion of vanillic acid to guaiacol by vanillate decarboxylase followed by the biotransformation of guaiacol to catechol by the aromatic ring$O$-demethylase $[38,39]$. Interestingly, experiments of a single-carbon source growth of strain SP-35 showed that when $0.5 \mathrm{~g} / \mathrm{L}$ aromatic compound was added as a carbon source, the ferulic acid, vanillin, vanillic acid and protocatechuate were exhausted at $48,72,36$, and $24 \mathrm{~h}$, respectively (Table 3 ). The fact that the conversion of vanillin to vanillic acid needed a longer time suggests that this is the rate-limiting step in the ferulic acid decomposition pathway. In addition, there is another biosynthesis pathway for vanillin in bacteria that is obtained by the conversion of $\rho$-coumaric acid via two intermediates [40]. However, there were no identified enzymes involved in the $\rho$-coumaric acid pathway in the genome of strain SP-35, and $\rho$-coumaric acid cannot be effectively used as the sole carbon source to support cell growth (Table 3). Our results indicate that the vanillin was mainly obtained from the ferulic acid pathway and the $\beta$-aryl ether pathways in the strain SP-35.

SA is the only metabolite identified in the MWL culture that belongs to the $\mathrm{S}$ type. The proposed pathway for the metabolism of SA starts with the conversion of SA to syringate by ALDH and the subsequent conversion of syringate to 3-O-methylgallate (3MGA) by vanillate/ syringate $O$-demethylase $(\mathrm{V} / \mathrm{SODM})$. The compound 3MGA was able to be biotransformed to gallate by V/ SODM. Four ALDH genes were observed in the genome of strain SP-35, among them, one is involved in the degradation of $\mathrm{SA}$, which needs to be verified in the future. The degradation of syringate in Sphingomonas paucimobilis SYK-6 involves the participation of two V/SODM, LigM and desA. The substrate of LigM is 3MGA and the substrate of desA is syringate [41]. Correspondingly, there were also two V/SODM-encoding genes in strain SP-35 that were identified, which may have encoded the enzyme that degrades syringate and the enzyme that degrades 3MGA, respectively. In addition, 3MGA was able to be degraded through multiple pathways and was able to be converted into different products, including 2-pyrone-4,6-dicarboxylate and 4-carboxy-2-hydroxy6-methoxy-6-oxohexa-2,4-dienoate via protocatechuate 4, 5-dioxygenase and 3MGA 3, 4-dioxygenase [42, 43]. However, the genes encoding protocatechuate 4, 5-dioxygenase or 3MGA 3, 4-dioxygenase still have not been identified in strain SP-35. Notably, ALDH has a wide range of substrates and is able to degrade benzaldehyde and its derivatives, such as SA, vanillin, benzaldehyde, $p$-hydroxybenzaldehyde, protocatechualdehyde, $m$-anisaldehyde, veratraldehyde, coniferyl aldehyde, salicylaldehyde, $m$-hydroxybenzaldehyde, etc. [44]. Therefore, the extensiveness of the enzyme's possible substrates has led to difficulties in elucidating the metabolic pathways of lignin-derived aromatic compounds, and the diversity of lignin degradation intermediates has also increased the complexity of the lignin metabolism network in bacteria.

Enzymes involved in lignin degradation are quite different between strain SP-35 and fungi. For example, lignin-modifying enzymes produced by fungus including Lacc, LiP, MnP, VP and DyPs, while the strain SP-35 only include Lacc and DyPs. Lignin-degrading auxiliary enzymes produced by fungus including glyoxal oxidase, aryl alcohol oxidases, pyranose 2-oxidase, cellobiose dehydrogenase and glucose oxidase, but all above enzymes in the strain SP-35 were not found [45]. Usually, hydrogen peroxide was necessary for lignin degradation by LiP, MnP and VP of fungus, but DyP from bacteria was proved able to degrade lignin without hydrogen peroxide [46].

\section{Conclusions}

In this report, we described a detailed study of the degradation of MWL and AL by the strain SP-35, which could be an efficient cell factory for the conversion of lignin to high-value aromatic compounds. In summary, there are at least five enzymes in strain SP-35 involved in the oxidation and depolymerization of lignin including DyPs, and Lacc, etc. The variations of absorption of FT-IR spectra indicate that the degradation of MWL by strain SP-35 included the oxidative cleavage of the lignin aromatic ring structures and the modification of its side chains. Based on genome analysis and the intermediate metabolites, we speculated multiple aromatic compounds' metabolic pathways in strain SP-35, including benzoate, phenylacetic acid, 4-hydroxycinnamic acid, 4-hydroxyacetophenone, 3, 4-dihydroxyphenylglycol, ferulic acid, and syringaldehyde, and a DPHG degradation 
pathway first identified in bacteria, etc. In addition, the $\beta$-aryl ether cleavage pathway was first described in bacteria other than the family Sphingomonadaceae. A total of 94 genes were identified to encode 34 enzymes and 5 regulatory factors involved in lignin degradation and catabolism of its intermediates. It was suggested that the degradation of lignin constitutes a complex metabolic network in strain SP-35.

The large-scale production of value-added aromatic compounds released by SP-35, such as vanillin, SA, coumaric acid, catechol and protocatechuic acid is fascinating if the bottleneck of genetic manipulation was tackled. Therefore, the future work will focus on inactivation of vanillin dehydrogenase, aldehyde dehydrogenase, CatA and PcaA enzyme activities by gene knockout gene editing or other synthetic biology methods, thereby blocking the metabolic pathway of vanillin, SA, coumaric acid, $\beta$-KAP achieves the purpose of accumulating high-value aromatic compounds on large-scale.

\section{Additional files}

Additional file 1. The tolerance of strain SP-35 cultrue for aromatic compounds.

Additional file 2. Genome annotation of Comamonas serinivorans SP-35.

\section{Abbreviations}

MWL: milled wood lignin; AL: alkali lignin; H: $\rho$-hydroxyphenyl; G: guaiacyl; S: syringyl; LiP: lignin peroxidases; MnP: manganese peroxidases; VP: versatile peroxidases; DyPs: dye decolorizing peroxidases; Lacc: laccases; $\beta$-KAP: $\beta$-ketoadipate pathway; LB: Luria broth; LM: lignin medium; COD: chemical oxygen demand; SEM: scanning electron microscopy; FTIR: Fourier-transform infrared spectrometer; GC-MS: gas chromatography-mass spectrometry; BSTFA: N, O-bis (trimethylsilyl) trifluoroacetamide; TMCS: trimethylchlorosilane; TMS: trimethylsilyl; SMRT: single molecule real time; HCA: 4-hydroxycinnamic acid; 2,4-DHB: 2,4-dihydroxybenzaldehyde; HAP: 4'-hydroxyacetophenone; DHPG: 3,4-dihydroxyphenylglycol; HBA: 4-hydroxybenzoic acid; DOMA: 3,4-dihydroxymandelic acid; 3-MP: 3-methylphenol; BHT: butylated hydroxytoluene; GSTs: glutathione transferases; NAD-ADH: NAD-dependent alcohol dehydrogenase; GGE: guaiacylglycerol- $\beta$-guaiacyl ether; MPHPV: $\alpha$-(2-methoxyphenoxy)- $\beta$-hydroxypropiovanillone; GS-HPV: generateß-glutathionyl-y-hydroxypropiovanillone; GSSG: glutathione disulfide; HPV: $\gamma$-hydroxypropiovanillone; WA: vanilloyl acetic acid; PA: phenylacetic acid; HBAld: 4-hydroxybenzaldehyde; SA: syringaldehyde; catA: catechol 1, 2-dioxygenase; catB: muconate cycloisomerase; catC: muconolactone D-isomerase; pcaD: $\beta$-ketoadipate enol-lactone hydrolase; pcal: 3-oxoadipate CoA-transferase subunit A; pcaJ: 3-oxoadipate CoA-transferase subunit B; pcaF: $\beta$-ketoadipyl CoA thiolase; pcaC: carboxymuconolactone decarboxylase; pcaHG: protocatechuate 3, 4-dioxygenase; pcaB: $\beta$-carboxymuconate cycloisomerase; pcaR: $\beta$-KAP transcription regulator; DHCDC: cis-1, 2-dihydroxycyclohexa-3, 5-diene-1-carboxylate; BadR: benzoate anaerobic degradation regulator; PA-CoA: phenylacetyl-CoA; ep-CoA: 1, 2-epoxyphenylacetyl-CoA; DHAP: 2, 4'-dihydroxyacetophenone; HBA-CoA: 4-hydroxybenzoyl-CoA; ALDH: aldehyde dehydrogenase; HPA: 4-hydroxyphenylacetate; HAPMO: 4-hydroxyacetophenone monooxygenase; HPCA: 3,4-dihydroxyphenylacetate; ADH: alcohol dehydrogenase; MHPG: 3-methoxy-4-hydroxyphenylglycol; COMT: catechol O-methyltransferase; MOPEGAL: 3-methoxy-4-hydroxyphenylglycolaIdehyde; VMA: 3-methoxy-4-hydroxymandelate; 3MGA: 3-O-methylgallate; V/ SODM: vanillate/syringate O-demethylase.

\section{Authors' contributions}

DCZ and JZS designed the experiments, DCZ, HBS, PPZ, MZ and ALG performed the experiments. DCZ, WMZ and BY and WJQ analyzed data. DCZ and JZS supervised the experiments. DCZ wrote the manuscript. All authors read and approved of the final manuscript.

\section{Author details}

${ }^{1}$ Biofuels Institute, School of the Environment and Safety Engineering, Jiangsu University, Zhenjiang, Jiangsu, China. ${ }^{2}$ State Key Laboratory of Applied Microbiology Southern China, Guangdong Provincial Key Laboratory of Microbial Culture Collection and Application, Guangdong Open Laboratory of Applied Microbiology, Guangdong Institute of Microbiology, Guangzhou, China.

${ }^{3}$ Bioproducts, Sciences and Engineering Laboratory, Department of Biological Systems Engineering, Washington State University, Richland, WA 99354, USA.

${ }^{4}$ Biological Sciences Division and Environmental Molecular Sciences Laboratory, Pacific Northwest National Laboratory, Richland, WA 99352, USA.

\section{Acknowledgements}

The Authors thanks Pacific Northwest National Laboratory and the Bioproducts, Science \& Engineering Laboratory and Department of Biological Systems Engineering at Washington State University for support of part of work.

\section{Competing of interests}

The authors declare that they have no competing of interests.

\section{Availability of data and materials}

Data supporting the findings can be found at Jiangsu University, School of Environmental and Safety Engineering, Zhenjiang, China.

\section{Consent for publication}

Not applicable.

\section{Ethical approval and consent to participate}

Not applicable.

\section{Funding}

This work was supported by the National Natural Science Foundation of China (Grant No. 31772529); the Grant Key Project of Science and Technology Program of Jiangsu Province (BE2016353), China; and U.S. Department of Energy DOE EERE Awards (DE-EE0007104 and DE-EE0006112) with the Bioproducts, Science \& Engineering Laboratory and Department of Biological Systems Engineering at Washington State University.

\section{Publisher's Note}

Springer Nature remains neutral with regard to jurisdictional claims in published maps and institutional affiliations.

Received: 1 July 2018 Accepted: 15 December 2018 Published online: 27 December 2018

\section{References}

1. Nelsen MP, DiMichele WA, Peters SE, Boyce CK. Delayed fungal evolution did not cause the Paleozoic peak in coal production. Proc Natl Acad Sci U S A. 2016;113:2442-7.

2. Ramachandra M, Crawford DL, Hertel G. Characterization of an extracellular lignin peroxidase of the lignocellulolytic actinomycete Streptomyces viridosporus. Appl Environ Microbiol. 1988;54:3057-63.

3. Brown ME, Walker MC, Nakashige TG, lavarone AT, Chang MC. Discovery and characterization of heme enzymes from unsequenced bacteria: application to microbial lignin degradation. J Am Chem Soc. 2011;133:18006-9.

4. Zhu D, Zhang P, Xie C, Zhang W, Sun J, Qian WJ, Yang B. Biodegradation of alkaline lignin by Bacillus ligniniphilus L1. Biotechnol Biofuels. 2017;10:44.

5. Masai E, Katayama Y, Fukuda M. Genetic and biochemical investigations on bacterial catabolic pathways for lignin-derived aromatic compounds. Biosci Biotechnol Biochem. 2007;71:1-15.

6. Pereira JH, Heins RA, Gall DL, MCAndrew RP, Deng K, Holland KC, Donohue TJ, Noguera DR, Simmons BA, Sale KL, et al. Structural and 
biochemical characterization of the early and late enzymes in the lignin beta-aryl ether cleavage pathway from Sphingobium sp. SYK-6. J Biol Chem. 2016;291:10228-38.

7. Kamimura N, Takahashi K, Mori K, Araki T, Fujita M, Higuchi Y, Masai E. Bacterial catabolism of lignin-derived aromatics: new findings in a recent decade: update on bacterial lignin catabolism. Environ Microbiol Rep. 2017:9:679-705.

8. Wells T Jr, Ragauskas AJ. Biotechnological opportunities with the betaketoadipate pathway. Trends Biotechnol. 2012;30:627-37.

9. Zakzeski J, Bruijnincx PC, Jongerius AL, Weckhuysen BM. The catalytic valorization of lignin for the production of renewable chemicals. Chem Rev. 2010;110:3552-99

10. Gronqvist S, Viikari L, Niku-Paavola ML, Orlandi M, Canevali C, Buchert J. Oxidation of milled wood lignin with laccase, tyrosinase and horseradish peroxidase. Appl Microbiol Biotechnol. 2005;67:489-94.

11. Holtman KM, Chang HM, Kadla JF. Solution-state nuclear magnetic resonance study of the similarities between milled wood lignin and cellulolytic enzyme lignin. J Agric Food Chem. 2004;52:720-6.

12. Zhu D, Xie C, Huang Y, Sun J, Zhang W. Description of Comamonas serinivorans sp. nov., isolated from wheat straw compost. Int J Syst Evol Microbiol. 2014;64:4141-6.

13. Bu LX, Tang Y, Gao YX, Jian HL, Jiang JX. Comparative characterization of milled wood lignin from furfural residues and corncob. Chem Eng J. 2011;175:176-84.

14. Eaton AD, Clesceri LS, Greenberg AE. Standard methods for the examination of water and wastewater. Washington, D.C: American Public Health Association; 1995.

15. Chin CS, Alexander DH, Marks P, Klammer AA, Drake J, Heiner C, Clum A, Copeland A, Huddleston J, Eichler EE, et al. Nonhybrid, finished microbial genome assemblies from long-read SMRT sequencing data. Nat Methods. 2013:10:563-9.

16. Zhang PP, Sun JZ, Xie CX, Zhu DC. Lignin degradation by Comamonas serinivorans C35. Microbiol China. 2017:44:1131-7.

17. Yuan TQ, He J, Xu F, Sun RC. Fractionation and physico-chemical analysis of degraded lignins from the black liquor of Eucalyptus pellita KP-AQ pulping. Polym Degrad Stab. 2009;94:1142-50.

18. Holtman KM, Chang HM, Jameel H, Kadla JF. Quantitative C-13 NMR characterization of milled wood lignins isolated by different milling techniques. J Wood Chem Technol. 2006;26:21-34.

19. Hofrichter $M, U$ Ulrich $R$, Pecyna $M J$, Liers $C$, Lundell T. New and classic families of secreted fungal heme peroxidases. Appl Microbiol Biotechnol. 2010:87:871-97.

20. Liers C, Pecyna MJ, Kellner H, Worrich A, Zorn H, Steffen KT, Hofrichter M, Ullrich R. Substrate oxidation by dye-decolorizing peroxidases (DyPs) from wood- and litter-degrading agaricomycetes compared to other fungal and plant heme-peroxidases. Appl Microbiol Biotechnol. 2013;97:5839-49.

21. Brzostowicz PC, Reams AB, ClarkTJ, Neidle EL. Transcriptional crossregulation of the catechol and protocatechuate branches of the betaketoadipate pathway contributes to carbon source-dependent expression of the Acinetobacter sp. strain ADP1 pobA gene. Appl Environ Microbiol. 2003:69:1598-606.

22. Romero-Steiner S, Parales RE, Harwood CS, Houghton JE. Characterization of the pcaR regulatory gene from Pseudomonas putida, which is required for the complete degradation of $p$-hydroxybenzoate. J Bacteriol. 1994:176:5771-9.

23. Valderrama JA, Durante-Rodriguez G, Blazquez B, Garcia JL, Carmona M, Diaz E. Bacterial degradation of benzoate: cross-regulation between aerobic and anaerobic pathways. J Biol Chem. 2012;287:10494-508.

24. Wright JD. Fungal degradation of benzoic acid and related compounds. World J Microbiol Biotechnol. 1993:9:9-16.

25. Nishikawa Y, Yasumi Y, Noguchi S, Sakamoto H, Nikawa J. Functional analyses of Pseudomonas putida benzoate transporters expressed in the yeast Saccharomyces cerevisiae. Biosci Biotechnol Biochem. 2008;72:2034-8.

26. Hirakawa H, Hirakawa Y, Greenberg EP, Harwood CS. BadR and BadM proteins transcriptionally regulate two operons needed for anaerobic benzoate degradation by Rhodopseudomonas palustris. Appl Environ Microbiol. 2015;81:4253-62.

27. Chen DW, Zhang Y, Jiang CY, Liu SJ. Benzoate metabolism intermediate benzoyl coenzyme $A$ affects gentisate pathway regulation in Comamonas testosteroni. Appl Environ Microbiol. 2014;80:4051-62.

28. Boll M, Loffler C, Morris BE, Kung JW. Anaerobic degradation of homocyclic aromatic compounds via arylcarboxyl-coenzyme A esters: organisms, strategies and key enzymes. Environ Microbiol. 2014;16:612-27.

29. Briganti F, Pessione E, Giunta C, Scozzafava A. Purification, biochemical properties and substrate specificity of a catechol 1,2-dioxygenase from a phenol degrading Acinetobacter radioresistens. FEBS Lett. 1997;416:61-4.

30. Nogales J, Macchi R, Franchi F, Barzaghi D, Fernandez C, Garcia JL, Bertoni G, Diaz E. Characterization of the last step of the aerobic phenylacetic acid degradation pathway. Microbiology. 2007;153:357-65.

31. O'Connor KE, O'Leary NP, Marchesi JR, Dobson AD, Duetz W. Isolation and characterization of a diverse group of phenylacetic acid degrading microorganisms from pristine soil. Chemosphere. 2005;61:965-73.

32. Loscher $R$, Heide L. Biosynthesis of $p$-Hydroxybenzoate from $p$-Coumarate and $p$-Coumaroyl-Coenzyme A in Cell-Free Extracts of Lithospermum erythrorhizon Cell Cultures. Plant Physiol. 1994;106:271-9.

33. Paria S, Halder P, Paine TK. Oxidative carbon-carbon bond cleavage of a alpha-hydroxy ketone by a functional model of 2,4'-dihydroxyacetophenone dioxygenase. Angew Chem Int Ed Engl. 2012;51:6195-9.

34. Kamerbeek NM, Moonen MJ, Van Der Ven JG, Van Berkel WJ, Fraaije MW, Janssen DB. 4-Hydroxyacetophenone monooxygenase from Pseudomonas fluorescens ACB. A novel flavoprotein catalyzing Baeyer-Villiger oxidation of aromatic compounds. Eur J Biochem. 2001;268:2547-57.

35. Rodriguez G, Lama A, Trujillo M, Espartero JL, Fernandez-Bolanos J. I solation of a powerful antioxidant from Olea europaea fruit-mill waste: 3,4-Dihydroxyphenylglycol. Lwt-Food Science and Technology. 2009;42:483-90.

36. Eisenhofer G, Kopin IJ, Goldstein DS. Catecholamine metabolism: a contemporary view with implications for physiology and medicine. Pharmacol Rev. 2004;56:331-49.

37. Siegrist J, Netzer J, Mordhorst S, Karst L, Gerhardt S, Einsle O, Richter M, Andexer JN. Functional and structural characterisation of a bacterial O-methyltransferase and factors determining regioselectivity. FEBS Lett. 2017:591:312-21.

38. Alvarez-Rodriguez ML, Belloch C, Villa M, Uruburu F, Larriba G, Coque JJ. Degradation of vanillic acid and production of guaiacol by microorganisms isolated from cork samples. FEMS Microbiol Lett. 2003;220:49-55.

39. Mallinson SJB, Machovina MM, Silveira RL, Garcia-Borras M, Gallup N, Johnson CW, Allen MD, Skaf MS, Crowley MF, Neidle EL, et al. A promiscuous cytochrome $\mathrm{P} 450$ aromatic O-demethylase for lignin bioconversion. Nat Commun. 2018;9:2487.

40. Walton NJ, Mayer MJ, Narbad A. Vanillin. Phytochemistry. 2003;63:505-15.

41. Abe T, Masai E, Miyauchi K, Katayama Y, Fukuda M. A tetrahydrofolatedependent O-demethylase, LigM, is crucial for catabolism of vanillate and syringate in Sphingomonas paucimobilis SYK-6. J Bacteriol. 2005;187:2030-7.

42. Kasai D, Masai E, Katayama Y, Fukuda M. Degradation of 3-O-methylgallate in Sphingomonas paucimobilis SYK-6 by pathways involving protocatechuate 4,5-dioxygenase. FEMS Microbiol Lett. 2007;274:323-8.

43. Kasai D, Masai E, Miyauchi K, Katayama Y, Fukuda M. Characterization of the 3-O-methylgallate dioxygenase gene and evidence of multiple 3-O-methylgallate catabolic pathways in Sphingomonas paucimobilis SYK-6.J Bacteriol. 2004:186:4951-9.

44. Kamimura N, Goto T, Takahashi K, Kasai D, Otsuka Y, Nakamura M, Katayama Y, Fukuda M, Masai E. A bacterial aromatic aldehyde dehydrogenase critical for the efficient catabolism of syringaldehyde. Sci Rep. 2017;7:44422.

45. Janusz G, Pawlik A, Sulej J, Swiderska-Burek U, Jarosz-Wilkolazka A, Paszczynski A. Lignin degradation: microorganisms, enzymes involved, genomes analysis and evolution. FEMS Microbiol Rev. 2017;41:941-62.

46. Ahmad M, Taylor CR, Pink D, Burton K, Eastwood D, Bending GD, Bugg TD. Development of novel assays for lignin degradation: comparative analysis of bacterial and fungal lignin degraders. Mol BioSyst. 2010;6:815-21. 